Europhys. Lett., 20 (6), pp. 559-564 (1992)

\title{
Phase Behaviour of Colloid + Polymer Mixtures.
}

\author{
H. N. W. Lekkerkerker (*), W. C.-K. Poon(**), P. N. Pusey (**) \\ A. Stroobants $(*)$ and P. B. Warren $(* * *)\left({ }^{\S}\right)$ \\ (*) Van't Hoff Laboratorium, Rijksuniversiteit te Utrecht \\ Padualaan 8, 3584 CH Utrecht, The Netherlands \\ (**) Department of Physics, The University of Edinburgh \\ Mayfield Road, Edinburgh, EH9 3JZ, UK \\ (***) Unilever Research, Port Sunlight Laboratory \\ Quarry Road East, Bebington, Wirral, L63 зJW, UK
}

(received 12 June 1992; accepted in final form 10 September 1992)

PACS. 82.70 - Disperse systems.

PACS. 64.75 - Solubility, segregation, and mixing.

\begin{abstract}
A new treatment of the phase behaviour of a colloid + nonadsorbing polymer mixture is described. The calculated phase diagrams show marked polymer partitioning between coexisting phases, an effect not considered in the usual effective-potential approaches to this problem. We also predict that under certain conditions an area of three-phase coexistence should appear in the phase diagram.
\end{abstract}

Introduction. - Phase separation in colloidal suspensions, induced by the addition of nonadsorbing polymer, is a phenomenon of fundamental interest and considerable technological importance. A theoretical explanation was first advanced by Asakura and Oosawa [1], and also independently by Vrij [2], based on the exclusion of polymer from the region between two colloid particles when their surface-surface separation becomes smaller than the diameter of a free polymer coil. The resulting imbalance in osmotic pressure gives rise to an effective attractive "depletion" force between the colloid particles [3,4]. At high enough concentration of polymer this depletion force causes the suspension to separate into colloid-poor and colloid-rich phases. In the latter the particles can, depending on conditions (see below), be in either liquidlike or crystalline spatial arrangements.

To predict the phase diagram of a colloid + polymer mixture, most workers to date have adopted an approach in which the depletion potential (an effective pair potential) is added to the parent interparticle potential; thermodynamic perturbation theory is then used to calculate phase stability boundaries [5,6]. Although experimental studies [6,7] show qualitative agreement with the predictions of these calculations, an important reservation UK.

$\left(^{\S}\right)$ Also at University of Edinburgh, Department of Physics, Mayfield Road, Edinburgh, EH9 3JZ, 
has been expressed recently [8-11]. The effective potential approach assumes that the polymer concentration is the same in coexisting phases and does not allow for the occurrence of polymer partitioning between the various phases [12,13].

Here we explore the consequences of a simple statistical mechanical model $[9,10]\left({ }^{1}\right)$ for a colloid + polymer mixture which can account for and predict polymer partitioning at phase separation. In the simplest approximation the polymer is supposed dilute and at its $\theta$-point, and the colloid particles interact as hard spheres. The only effect of the interaction between colloid and polymer is to restrict the volume in which the latter can move. Thus we treat the polymer as a suspension of freely interpenetrable coils whose centres of mass cannot approach closer than a distance $\vdots$ from any (nonadsorbing) surface. The centre of mass of a polymer is therefore excluded from a sphere of radius $a+i$ around a colloid particle of radius $a$. Here we show that a mean-field treatment of this model does indeed predict marked partitioning of the polymer. Furthermore, under certain conditions, we find a three-phase region in the phase diagram where colloidal gas, liquid and crystal coexist. Earlier treatments of the model only considered gas-liquid coexistence $[9,10]$.

Statistical mechanics. - A convenient starting point in the calculation of the free energy in our model is to work in the grand canonical ensemble $[4,10]$. The integration over the translational-polymer degrees of freedom may be performed exactly, resulting in an effective interaction between colloid particles of the form

$$
W=U_{\mathrm{C}}-\Pi_{\mathrm{P}}\left(\mu_{\mathrm{P}}\right) V_{\text {free }}\left(\boldsymbol{r}_{\mathrm{C}}\right) .
$$

In this expression $U_{\mathrm{C}}$ is the bare colloid interaction potential, $\Pi_{\mathrm{P}}$ is the osmotic pressure of a pure polymer system expressed in terms of the polymer chemical potential $\mu_{\mathrm{P}}$, and $V_{\text {free }}$ is the free volume in which the polymer coils can move. The free volume depends on the colloid particle positions, collectively denoted by $\boldsymbol{r}_{\mathrm{C}}$. The effective potential $W$ has a many-body nature, since a full expression for $V_{\text {free }}$ can be written only in terms of the mutual overlap of the excluded-volume shells of all the colloid particles. The meaning of the final term in eq. (1) is clearly the following: if the free volume changes due to a change in the positions of the colloid particles, work has to be done against the pressure exerted by the polymer molecules.

To make progress, we use a van der Waals mean field-approximation and replace $V_{\text {free }}\left(\boldsymbol{r}_{\mathrm{C}}\right)$ by its average value in the corresponding unperturbed system of colloidal particles [15]. Thus we write $V_{\text {free }}=\alpha V$, where the "free-volume fraction" $\alpha$ depends only on the colloid volume fraction $\phi=4 \pi a^{3} N_{\mathrm{C}} / 3 \mathrm{~V}$, and on the ratio $\delta / a$. The integration over the colloid degrees of freedom may now be performed, since the final term in (1) no longer contains any dependence on the colloid particle positions. Since colloidal systems are, to a good approximation, constant-volume systems [16], we present our results in terms of the Helmholtz free energy. The final expression, for the Helmholtz free energy of a system of $N_{\mathrm{C}}$ colloid particles and $N_{\mathrm{P}}$ polymer coils in a volume $V$, can be written in the suggestive form

$$
F=F_{\mathrm{C}}\left(N_{\mathrm{C}}, V\right)+F_{\mathrm{P}}\left(N_{\mathrm{P}}, \alpha V\right) .
$$

The free energy decouples into a term corresponding to pure colloid in a volume $V$, and a term corresponding to pure polymer in a volume $\alpha V$. The interaction between polymer and colloid is contained solely in the dependence of $\alpha$ on the colloid volume fraction.

( $\left.{ }^{1}\right)$ We mention that this model corresponds to the asymmetric, nonadditive hard-sphere mixture introduced some years ago in the context of liquid-vapour transitions [14]. 
Since we treat the polymer as a suspension of noninteracting coils, we can write

$$
F_{\mathrm{P}}\left(N_{\mathrm{P}}, \alpha V\right)=k T n V \log \frac{n}{\alpha}+\text { other terms, }
$$

where $n=N_{\mathrm{P}} / V$. The other terms in (3) are linear in $N_{\mathrm{P}}$ and $V$ and do not contribute to the determination of the phase behaviour. The colloid contribution to (2) can be derived by integration from the hard-sphere equation of state

$$
F_{\mathrm{C}}\left(N_{\mathrm{C}}, V\right)=\frac{3 k T}{4 \pi a^{3}} V \phi \int \frac{Z}{\phi} \mathrm{d} \phi,
$$

where $Z(\phi)$ is the hard-sphere compressibility. For colloidal-fluid phases we use the expression for $Z$ suggested by Carnahan and Starling [17]; for colloidal crystals (FCC lattice) we use that suggested by Hall [18].

The chemical potentials of the colloid and polymer $\left(\mu_{\mathrm{C}}\right.$ and $\left.\mu_{\mathrm{P}}\right)$, and the total osmotic pressure $(I)$ are now obtained from the free energy by differentiation:

$$
\begin{gathered}
\left(\mu_{\mathrm{C}}-\mu_{\mathrm{C}}^{0}\right) / k T=\int \frac{Z}{\phi} \mathrm{d} \phi+Z-\frac{4}{3} \pi a^{3} \frac{n}{\alpha} \frac{\mathrm{d} \alpha}{\mathrm{d} \phi}, \\
\left(\mu_{\mathrm{P}}-\mu_{\mathrm{P}}^{0}\right) / k T=\log \frac{n}{\alpha}, \\
4 \pi a^{3} \Pi / 3 k T=\phi Z+\frac{4}{3} \pi a^{3} \frac{n}{\alpha}\left(\alpha-\phi \frac{\mathrm{d} \alpha}{\mathrm{d} \phi}\right),
\end{gathered}
$$

where $\mu_{\mathrm{C}}^{0}$ and $\mu_{\mathrm{P}}^{0}$ are reference chemical potentials. The absolute value of the integration constant in (5) is not required. However, we can fix $\mu_{C}$ (fluid) relative to $\mu_{C}$ (solid) by equating them at the expected coexistence compositions in the absence of polymer, namely $\phi($ fluid $)=0.494, \phi($ solid $)=0.545[19]$.

To use these expressions, we need the free-volume fraction $\alpha(\phi)$. An approximate expression for $\alpha$ can be obtained by realising that, according to Widom's particle insertion method [15], the chemical potential of a test hard-sphere species $Q$ of radius $\delta$ and volume fraction $\phi_{Q} \rightarrow 0$ in a sea of hard spheres of radius $a$ and volume fraction $\phi$ can be written as

$$
\mu_{Q}=\mu_{Q}^{0}+k T \log \phi_{Q}-k T \log \alpha,
$$

where $\alpha$ is exactly the desired free-volume fraction [10]. Comparing this expression with the well-known scaled particle expression [20], or equivalently the Percus-Yevick result [21], for the chemical potentials of a mixture of hard spheres of the kind indicated, one obtains

$$
\alpha=(1-\phi) \exp \left[-A \gamma-B \gamma^{2}-C \gamma^{3}\right]
$$

in which $\gamma=\phi /(1-\phi), A=3 \xi+3 \xi^{2}+\xi^{3}, B=9 \xi^{2} / 2+3 \xi^{3}$, and $C=3 \xi^{3}$ (we have set $\xi=\delta / a$ ). We use the same expression for both the fluid and the solid phases $\left({ }^{2}\right)$.

The calculation of the phase behaviour is facilitated by eliminating $\mu_{\mathrm{P}}$ between eqs. (5)-(7).

$\left({ }^{2}\right)$ The accuracy of expression (9) in the dense fluid and the solid is currently being evaluated and will be the subject of a fortheoming publication. 
Defining $n_{\mathrm{R}}=n / \alpha$, eq. (6) gives

$$
n_{\mathrm{R}}=\exp \left[\left(\mu_{\mathrm{P}}-\mu_{\mathrm{P}}^{0}\right) / k T\right]
$$

and we obtain expressions for $\mu_{\mathrm{C}}$ and $\Pi$ which are functions of $\phi$ and $n_{\mathrm{R}}$. Now, $n_{\mathrm{R}}$ is a function of polymer chemical potential and is therefore the same in coexisting phases. Thus to calculate the compositions $\phi_{1}$ and $\phi_{2}$ of a pair of such phases, we only need to solve $\mu_{\mathrm{C}}\left(\phi_{1}, n_{\mathrm{R}}\right)=\mu_{\mathrm{C}}\left(\phi_{2}, n_{\mathrm{R}}\right)$ and $\Pi\left(\phi_{1}, n_{\mathrm{R}}\right)=\Pi\left(\phi_{2}, n_{\mathrm{R}}\right)$, at given $n_{\mathrm{R}}$. The polymer concentrations in these phases are then found from $n_{1}=\alpha\left(\phi_{1}\right) n_{\mathrm{R}}$ and $n_{2}=\alpha\left(\phi_{2}\right) n_{\mathrm{R}}$. In fact $n_{\mathrm{R}}$ can be interpreted as the polymer concentration in a reservoir of pure polymer in equilibrium with the system, thus providing a connection with previous work [10].

Results. - Phase diagrams in the $\left(\phi, n_{\mathrm{R}}\right)$-plane calculated by this method are shown in fig. 1 for $\% / a=0.1$ and 0.4 . With no added polymer $\left(n_{\mathrm{R}}=0\right)$ we recover the hard-sphere freezing transition, with freezing at $\phi \approx 0.50$ and melting at $\phi \approx 0.55$. For $\delta / a \leqslant 0.32$ the region of liquid-crystal coexistence is broadened strongly on addition of polymer (fig. 1a)). For $\delta / a \geqslant 0.32$, critical and triple points emerge in the phase diagram (fig. $1 b)$ ), which becomes analogous to that of a simple atomic material $[5,6,11]$. The results of fig. 1 look very similar to those obtained by the effective-potential method [5,6], with the important difference that the vertical axis in our diagrams is the concentration of polymer in a (hypothetical) reservoir of pure polymer in equilibrium with the system and not the polymer concentration in the system itself.

Since $n=\alpha n_{\mathrm{R}}$, phase diagrams in the experimentally accessible $(\phi, n)$-plane, shown in fig. 2, are obtained from those of fig. 1 literally by multiplication by $\alpha(\phi)$. For $\delta / a \leqslant 0.32$ we find simple fluid-solid coexistence, but with marked partitioning of polymer between the phases (fig. $2 a$ )). For $\delta / a \geqslant 0.32$ we find that the triple point of fig. $1 b$ ) expands into a region
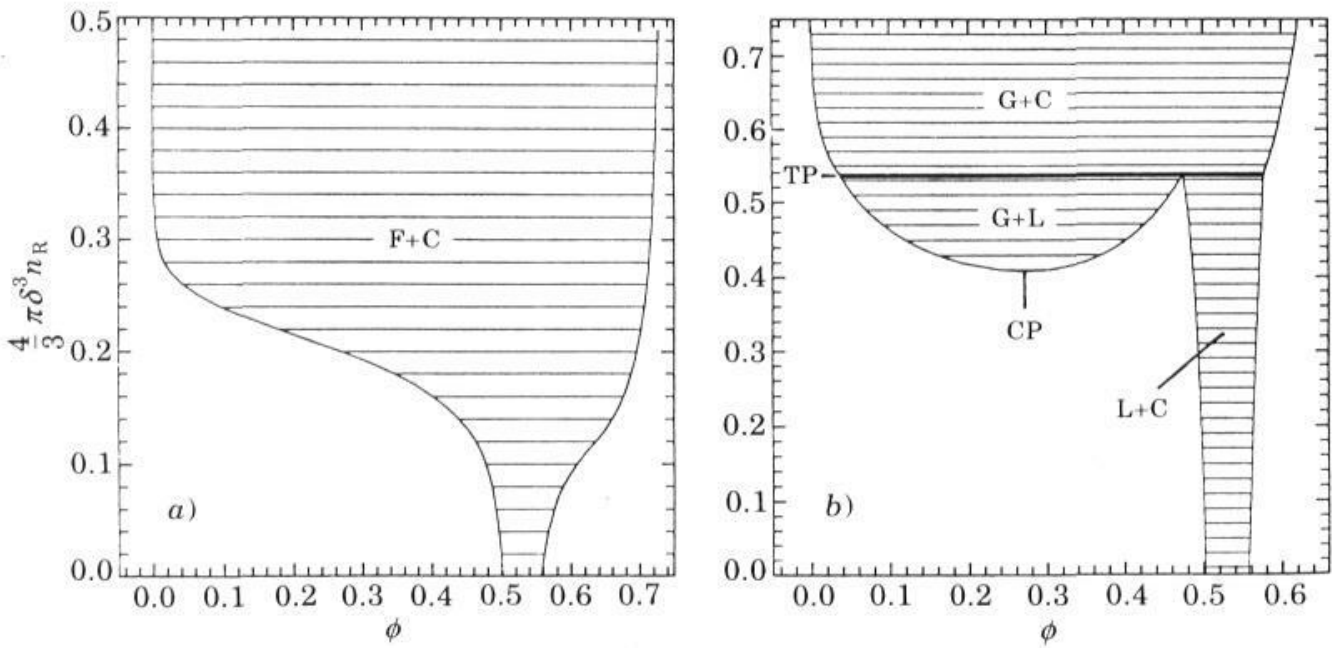

Fig. 1. - Phase diagrams of colloid-polymer mixtures showing colloid volume fraction $\phi$ against dimensionless polymer reservoir concentration $(4 / 3) \pi c^{3} n_{\mathrm{R}}$, for $\left.\% / a=0.1(a)\right)$ and $0.4(b)$ ). Tie lines are shown in the two-phase regions, whose composition is indicated as colloidal fluid (F), colloidal gas (G), colloidal liquid (L) or colloidal crystal (C). In $b$ ) the critical point (CP) and triple point (bold line marked TP) are also indicated. 

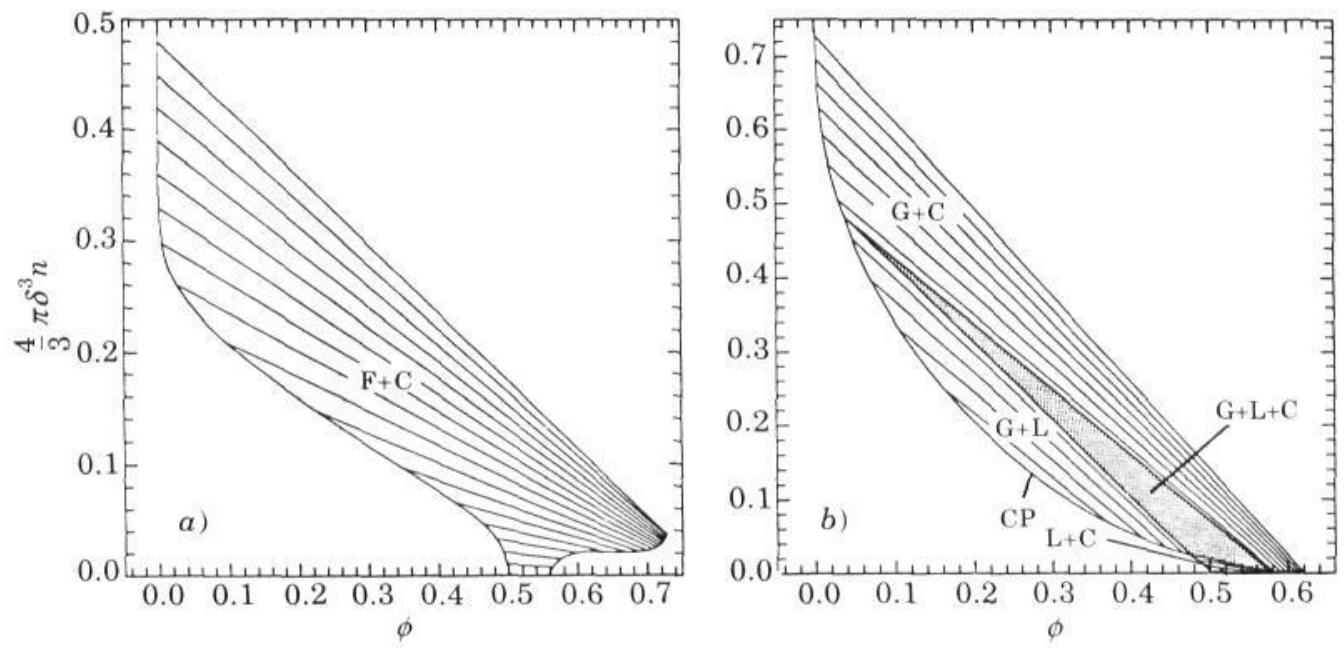

Fig. 2. - Phase diagrams of colloid-polymer mixtures as functions of colloid volume fraction $\phi$ against dimensionless polymer concentration $(4 / 3) \pi 0^{3} n$. The notation is the same as in fig. 1 . Note that the oblique tie lines imply considerable partitioning of the polymer among the phases. In the $(\phi, n)$-plane the triple point observed in $b$ ) becomes the region of three-phase coexistence (gas-liquid-crystal) shown shaded in $b$ ).

of three-phase coexistence (gas-liquid-solid) bounded by three distinct two-phase regions (fig. $2 b))\left({ }^{3}\right)$.

In fig. 1 and 2 tie lines are indicated in the two-phase regions. Because of mass conservation they are straight and the relative volumes of each phase can be obtained by the usual lever rule [16]. In the three-phase region of fig. $2 b$ ) the compositions of the phases are given by the vertices of the triangle and the phase volumes by an area rule described by Bartlett [16] in the context of binary mixtures of hard spheres with additive diameters.

Discussion. - We have presented a simple model of the colloid + nonadsorbing polymer system, in which the translational degrees of freedom of the polymer are treated on the same footing as those of the colloid. We predict marked polymer partitioning between coexisting phases, and the emergence of a three-phase region for $\delta / a \geqslant 0.32$. Whilst we have made a number of simplifying assumptions, we expect that these features will be preserved in a more realistic treatment. For instance at polymer concentrations approaching coil overlap $\left((4 / 3) \pi 0^{3} n \sim 1\right.$, see fig. 2$)$ it is certainly not reasonable to treat the polymer as ideal. A better expression for the polymer free energy could then be used in (3). In addition a more realistic colloid interaction could be incorporated into (4).

Experimentally, the data of Patel and Russel [12] indicate strong polymer partitioning in a mixture of polystyrene colloids and dextran, whereas Robins [13] observed a weaker effect in an emulsion + polymer system. Three-phase coexistence in colloid-polymer mixtures has not yet been observed. However, Vincent et al. [6], in a study of mixtures of stearyl-grafted colloidal silica and polydimethylsiloxane, found two-phase regions of gas-liquid, liquid-solid

$\left({ }^{3}\right)$ Compare [22] where it is shown that the triple point on the conventional $p, T$ phase diagram of a simple substance becomes a triple region in a "density-density" (i.e. specific volume-specific energy) representation. 
and gas-solid coexistence. These observations, combined with the predictions reported here, suggest that this, and similar systems, should also show a region of three-phase coexistence. That such an observation has not yet been reported may result from slow kinetics, particularly of the liquid-solid separation, and the consequent persistence of metastable states.

\section{*** $*$}

We acknowledge fruitful discussions with P. Bartlett, D. Frenkel, M. Lal, E. J. MeiJer and I. D. Rовв. The possibility of three-phase coexistence in this system was also independently pointed out to PBW by D. Roux. This work was partly supported by NATO grant $132 / 84$.

\section{REFERENCES}

[1] Asakura S. and Oosawa F., J. Chem. Phys., 22 (1954) 1255.

[2] VRiJ A., Pure Appl. Chem., 48 (1976) 471.

[3] Asakura S. and Oosawa F., J. Polymer Sci., 33 (1958) 183; refinements to this force law have also been suggested: Fleer G. J. and Scheutuens J. M. H. M., Adv. Colloid Interface Sci., 16 (1982) 341; Shaw M. R. and Thirumalai D., Phys. Rev. A, 44 (1991) 4797.

[4] Meijer E. J. and Frenkel D., Phys. Rev. Lett., 67 (1991) 1110.

[5] Gast A. P., Hall C. K. and Russel W. B., J. Colloid Interface Sci., 96 (1983) 251.

[6] Vincent B. J., Edwards J., Emmett S. and Croot R., Colloid Surf., 31 (1988) 267.

[7] Sperry P. R., Hopfenberg H. B. and Thomas N. L., J. Colloid Interface Sci, 82 (1981) 62.

[8] Canessa E., Grimson M. J. and Silbert M., Mol. Phys., 67 (1989) 1153.

[9] Santore M. M., Russel W. B. and Prud'homme R. K., Macromolecules, 22 (1989) 1317.

[10] Lekkerkerker H. N. W., Colloid Surf., 51 (1990) 419.

[11] Pusey P. N., in Liquids, Freezing and the Glass Transition, edited by J. P. Hansen, D. Levesque and J. ZinN-Justin (Elsevier) 1991, Chapt. 10.

[12] Patel P. D. and Russel W. B., J. Colloid Interface Sci., 131 (1989) 192.

[13] Robins M. M., in Microemulsions and Emulsions in Foods, edited by M. EL-NoKaly and D. CoRnell, ACS Symposium Series, 448 (1991) Chapt. 17.

[14] Widom B. and Rowlinson J. S., J. Chem. Phys., 52 (1970) 1670.

[15] Widom B., J. Chem. Phys., 39 (1963) 2808.

[16] Bartlett P., J. Phys. Condens. Matter, 2 (1990) 4979.

[17] Carnahan N. F. and Starling K. E., J. Chem. Phys., 53 (1970) 600.

[18] Hall K. R., J. Chem. Phys., 57 (1972) 2252.

[19] Hoover W. G. and Ree F. H., J. Chem. Phys., 49 (1968) 3609.

[20] Reiss H., Frisch H. L. and Lebowitz J. L., J. Chem. Phys., 31 (1959) 369; Lebowitz J. L., Helfand E. and Praestgaard E., J. Chem. Phys., 43 (1965) 774.

[21] Lebowitz J. L. and Rowlinson J. S., J. Chem. Phys., 41 (1964) 133.

[22] Planck M., Treatise on Thermodynamics, 3rd edition (Dover) 1926, § 189. 